\title{
Eficiência de Inoculantes de Rizóbio na Nodulação de Alfafa em Solo de Cerrado Deise Ferreira Xavier ${ }^{1}$, Fernando Teixeira Gomes ${ }^{2}$, Francisco José da Silva Lédo Antônio Vander Pereira1, 3
}

\begin{abstract}
RESUMO - Em condições controladas, foi conduzido um experimento na Embrapa Gado de Leite, Juiz de Fora, MG, com o objetivo de estudar a eficiência de dois inoculantes de Rhizobium meliloti comerciais no desenvolvimento de cultivares de alfafa em solo classificado como Latossolo Vermelho-Escuro, distrófico, fase cerrado. O delineamento experimental foi o de blocos ao acaso, em esquema fatorial 3x4, com quatro repetições e com os seguintes tratamentos: a) Três cultivares de alfafa: Crioula, Crioula CNPGL 1 e P30; b) Dois inoculantes comerciais mais duas testemunhas (com e sem nitrogênio). Aos 60 dias após o plantio, foi feito um corte a $5 \mathrm{~cm}$, determinando-se a massa seca (MS) e N-total da parte aérea das plantas. Aos 90 dias coletou-se a planta inteira, medindo-se a MS da parte aérea e da raiz e o conteúdo de $\mathrm{N}$ da parte aérea. Foi verificada também a presença ou não de nódulos nas raízes. Os resultados de massa seca da parte aérea e da raiz e N-total da parte aérea indicaram que os dois inoculantes comerciais utilizados foram eficientes para os três cultivares de alfafa em solo de cerrado com o pH corrigido. Com relação ao parâmetro nodulação, as testemunhas sem e com nitrogênio não nodularam, indicando que, no solo trabalhado, não há presença de $R$. meliloti. Estas observações reforçam a necessidade de inocular a alfafa com rizóbio específico nas regiões tropicais.
\end{abstract}

Palavras-chave: leguminosa forrageira, Medicago sativa, nitrogênio, nodulação, Rhizobium meliloti

\section{Efficiency of Rhizobia Inoculants on Nodulation of Alfalfa in a "Cerrado" Soil}

ABSTRACT - An experiment was carried out under controlled conditions at the Embrapa Dairy Cattle, Juiz de Fora, MG, aiming to study the efficiency of two commercial inoculants of Rhizobium meliloti on the development of alfalfa cultivars in a "cerrado" soil classified as Dark-Red Latossol (Oxisol). The experimental design was a randomized blocks, in a 3 x 4 factorial, with four replications and the following treatments: a) three alfalfa cultivars: Crioula, Crioula CNPGL and P30; b) two commercial inoculants and two checks (with and without nitrogen). Sixty days after sowing, a harvest was performed at $5 \mathrm{~cm}$ height from ground level, to determine dry matter (DM) weight and total-N in the above ground parts. At ninety days, the whole plants were sampled, to measure the DM yield of both above ground parts and roots, and $\mathrm{N}$ content of above ground parts. The presence of nodules in the roots was examined. Results of DM yield of above ground parts and roots, and of total-N in the above ground parts, indicated that the two commercial inoculants used were efficient for all the three alfalfa cultivars in the soil, which had its $\mathrm{pH}$ corrected. Regarding nodulation, the checks with and without nitrogen did not nodulate, indicating that Rhizobium meliloti was not present in the soil. These observations reinforce the need of inoculation of alfalfa with appropriate rhizobia in tropical regions.

Key Words: forage legumes, Medicago sativa, nitrogen, nodulation, Rhizobium meliloti

\section{Introdução}

A alfafa (Medicago sativa L.) é considerada uma das mais importantes plantas forrageiras de clima temperado, reunindo algumas das mais desejadas características, como alto valor nutritivo, elevada produção de forragem, boa aceitabilidade e alta digestibilidade (Carvalho \& Vilela, 1994), sendo, por isso, indicada para alimentação de vacas de alta produção de leite. No Brasil, tem sido crescente o interesse por essa cultura, ocasionado, principalmente, pelo processo de intensificação dos sistemas de produção de leite nas regiões Sul, Sudeste e Centro-Oeste. Nestes sistemas, são utilizados animais de maior potencial genético, que exigem plantas forrageiras de melhor valor nutritivo (Pereira et al., 2001).

Pesquisas recentes conduzidas pela Embrapa Gado de Leite e outras Instituições de Pesquisa, têm demonstrado que a alfafa possui boa adaptação e alta produção de forragem em diferentes regiões do Brasil (Moreira et al., 1996; Botrel \& Alvim, 1997; Pereira et al., 1998; Ferreira et al., 1999; Viana et al.,1999; Botrel et al., 2000, 2001; Sales, 2001). Nesses trabalhos, foram avaliados cultivares de alfafa introduzidas

\footnotetext{
${ }^{1}$ Pesquisador da Embrapa Gado de Leite. Rua Eugênio do Nascimento, 610, Dom Bosco, CEP: 36038-330, Juiz de Fora, MG. E.mail: dfxavier@cnpgl.embrapa.br

2 Professor do Centro de Ensino Superior de Juiz de Fora - CES.

${ }^{3}$ Bolsista do CNPq.
} 
de outros países, juntamente com o cultivar Crioula, ou suas populações derivadas. De maneira geral, o cultivar Crioula e suas populações derivadas estiveram sempre entre os mais produtivos, com alguns cultivares introduzidos também apresentando bom desempenho, com destaque para P-30, originário da Argentina (Ferreira et al., 1999; Sales, 2001; Botrel et al., 2001).

Um dos fatores básicos que contribuem para a importância da alfafa como planta forrageira é sua capacidade de fixar o nitrogênio atmosférico, por meio da simbiose com a bactéria Rhizobium meliloti Dangeard. Estimativas desta fixação em clima temperado variam de 170 a $224 \mathrm{~kg} / \mathrm{ha} /$ ano (Heichel et al., 1984). Em condições tropicais, esta bactéria não é encontrada naturalmente nos solos recomendados para seu cultivo. Assim, não há nodulação da alfafa com as estirpes nativas, havendo sempre a necessidade de se inocular suas sementes com rizóbio específico (Franco, 1994).

Deve-se considerar também que a alfafa é bastante sensível à acidez do solo (Carvalho et al., 1994), sendo, portanto, importante a aplicação de calcário para maximizar a simbiose e a produtividade desta cultura (Franco, 1994). Na fase inicial de estabelecimento, a alfafa crescida em solo ácido produz menos nódulos que quando desenvolvida em solo com $\mathrm{pH} 7$ (Cheng et al., 2002).

Este trabalho foi desenvolvido com a finalidade se estudar a eficiência de dois inoculantes de alfafa comerciais disponíveis no mercado no desenvolvimento dos cultivares Crioula, Crioula CNPGL 1 e P-30, sob condições de solo de cerrado com $\mathrm{pH}$ corrigido.

\section{Material e Métodos}

O experimento foi conduzido em casa de vegetação na Embrapa Gado de Leite, em Juiz de Fora, MG, no período de 13 de setembro a 11 de dezembro de 2003. Foram avaliados três cultivares de alfafa (Crioula, Crioula CNPGL 1 e P-30), cultivados em vasos de polietileno rígido preenchidos com $3 \mathrm{dm}^{3}$ de solo, classificado como Latossolo Vermelho-Escuro, distrófico, fase cerrado, coletado em Sete Lagoas (MG) à profundidade de $0-20 \mathrm{~cm}$. O solo foi seco ao ar, passado em malha de $4 \mathrm{~mm}$ de diâmetro, misturado e homogeneizado. As características químicas do solo foram: $\mathrm{pH}$ em água (1:2,5), 5,3; M.O., $2,55 \mathrm{~g} / \mathrm{dm}^{3}$; cátions trocáveis $\left(\mathrm{cmol}_{\mathrm{c}} / \mathrm{dm}^{3}\right): \mathrm{Al}, 0,3 ; \mathrm{Ca}, 2,9 ; \mathrm{Mg}, 0,5$ e K, 0,08; P-disponível (Mehlich), 2,12 mg/kg.
Considerando-se que os três cultivares de alfafa testados foram selecionados sob solos com $\mathrm{pH}$ próximo à neutralidade e que a simbiose desta leguminosa com o $R$. meliloti é sensível à acidez, foi realizada a correção do $\mathrm{pH}$ do solo pelo método de saturação por bases com a aplicação de $\mathrm{CaCO}_{3}+\mathrm{MgCO}_{3}$, na relação $\mathrm{Ca}: \mathrm{Mg}=4: 1$ em equivalentes, correspondendo ao valor de neutralização (VN) de $1.000 \mathrm{~kg} / \mathrm{ha}$ de $\mathrm{CaCO}_{3}$ (Siqueira et al., 1980) em quantidades equivalentes a $4 \mathrm{t}$ de calcário/ha. Foi feita à aplicação do calcário por vaso, que, após a homogeneização, foram mantidos na capacidade de campo durante 60 dias antes do plantio, elevando o $\mathrm{pH}$ para 6,3. A adubação mineral foi realizada na véspera do plantio, com a adição de $75 \mathrm{mg} / \mathrm{dm}^{3}$ de $\mathrm{P}_{2} \mathrm{O}_{5}$ e 50 $\mathrm{mg} / \mathrm{dm}^{3}$ de $\mathrm{K}_{2} \mathrm{O}$. Foram aplicadas também os seguintes nutrientes por $\mathrm{dm}^{3}$ de solo: $150 \mathrm{mg}$ de $\mathrm{MgSO}_{4}$. $7 \mathrm{H}_{2} \mathrm{O} ; 15,8 \mathrm{mg}$ de $\mathrm{CuSO}_{4} .5 \mathrm{H}_{2} \mathrm{O} ; 8,91 \mathrm{mg}$ de $\mathrm{ZnSO}_{4}$ . $7 \mathrm{H}_{2} \mathrm{O} ; 0,30 \mathrm{mg}$ de $\mathrm{H}_{3} \mathrm{BO}_{3} ; 0,50 \mathrm{mg}$ de $\mathrm{Na}_{2} \mathrm{MoO}_{4}$. $2 \mathrm{H}_{2} \mathrm{O} ; 20,00 \mathrm{mg}$ de $\mathrm{FeSO}_{4} \cdot 7 \mathrm{H}_{2} \mathrm{O}$.

$\mathrm{O}$ delineamento experimental adotado foi o de blocos ao acaso, com quatro repetições, de modo que os tratamentos foram dispostos em esquema fatorial $3 \times 4$, combinando-se os três cultivares de alfafa: Crioula (Crioula CNPGL 1 e P-30) e os quatro tratamentos de inoculação (inoculante comercial 1; inoculante comercial 2; sem inoculante com adubação nitrogenada; e sem inoculante). Cada unidade experimental foi constituída de um vaso com quatro plantas.

Para os tratamentos com inoculação de Rhizobium meliloti, as sementes foram inoculadas conforme descrito por Xavier \& Carvalho (1985). O tratamento sem inoculação com adubação nitrogenada recebeu 5 $\mathrm{mg}$ de $\mathrm{N}$ por vaso, sete dias após a germinação, e 20 $\mathrm{mg}$ de $\mathrm{N}$ por vaso, quinzenalmente, até a colheita, na forma de $\mathrm{NH}_{4} \mathrm{NO}_{3}$. Em todos os tratamentos, a semeadura foi realizada com seis sementes, e após dez dias, foi efetuado o desbaste, deixando-se quatro plantas por vaso. Os vasos foram irrigados sempre que necessário, com água deionizada, para manter a umidade do solo próxima à capacidade de campo.

Aos 60 dias após o plantio, quando as cultivares apresentavam pelo menos $10 \%$ de florescimento (aparecimento das primeiras flores), foi feito o primeiro corte a $5 \mathrm{~cm}$. As plantas foram imediatamente pesadas, para determinação do peso da matéria fresca da parte aérea. Em seguida, foram acondicionadas em sacos de papel e colocadas em estufa com ventilação forçada a $65^{\circ} \mathrm{C}$, até peso constante, para determina- 
ção da matéria seca da parte aérea. Foi determinado também o teor de N (Bremner, 1965) na matéria seca da parte aérea. Aos 90 dias após o plantio, quando os cultivares apresentavam-se pelo menos $10 \%$ de florescimento, coletou-se a planta inteira, medindo-se a MS da parte aérea e da raiz e o conteúdo de $\mathrm{N}$ da parte aérea. Foi verificada a presença ou não de nódulos nas raízes, considerando-se que 90 dias de idade da alfafa sejam satisfatórios para os estudos de nodulação em vasos e em casa de vegetação. Embora tendo simulado o manejo de cortes, recomendado na produção de alfafa, é importante ressaltar a necessidade de um experimento de campo de longa duração, para validação dos resultados.

Os dados foram submetidos à análise estatística por intermédio do SAS. Para as características produção de massa seca da parte aérea e conteúdo de nitrogênio, a análise estatística foi realizada considerando-se um esquema de parcela subdividida no tempo (Ramalho et al., 2000). Neste caso, os tratamentos combinados em esquema fatorial $3 \times 4$ (três cultivares e quatro tratamentos) constituíram a parcela e os cortes, a subparcela.

\section{Resultados e Discussão}

$\mathrm{O}$ efeito de cultivar não-significativo $(\mathrm{P}<0,05)$ indica que os três cultivares de alfafa tiveram comportamento semelhante, o que pode ser atribuído à pouca variação entre os cultivares, uma vez que foram avaliados e selecionados como os melhores em experimentos anteriores sob as mesmas condições de solo e clima (Viana et al., 1999; Botrel et al., 2001), ou, ainda, ao fato de responderem de maneira semelhante, não havendo, portanto, interação genótipo x rizóbio.

Para as características conteúdo de nitrogênio total (N-Total) e produção de massa seca da raiz (MS da Raiz), avaliadas apenas no segundo corte, houve efeito significativo para os tratamentos de inoculação (TI) $(\mathrm{P}<0,05)$. Os efeitos de cultivares e da interação cultivares $\mathrm{x}$ TI foram não significativos $(\mathrm{P}>0,05)$. Considerando o N-total, os tratamentos com inoculantes 1 e 2 foram superiores aos tratamentos sem inoculante, com e sem adubação nitrogenada. Para massa seca da raiz, apenas o tratamento sem inoculante e sem nitrogênio ( $4,83 \mathrm{~g} / \mathrm{vaso})$ foi inferior aos tratamentos com inoculantes 1 e $2(6,81$ e $7,15 \mathrm{~g} /$ vaso, respectivamente).

As análises de variância para produção de massa
Tabela 1 - Efeito de dois inoculantes comerciais no desenvolvimento de alfafa, expressos em conteúdo de nitrogênio $(\mathrm{N})$ e massa seca $(\mathrm{MS})$ da raiz. Dados do segundo corte

Table 1 - Effect of two commercial inoculants on the development of alfalfa, expressed in terms of nitrogen content $(N)$ and dry matter (DM) of the roots. Data from second harvest

\begin{tabular}{lcc}
\hline $\begin{array}{l}\text { Tratamentos } \\
\text { Treatments }\end{array}$ & $\begin{array}{c}\text { N-total (g/vaso) } \\
\text { Total- } N(g / \text { pot })\end{array}$ & $\begin{array}{c}\text { MS da raiz }(\mathrm{g} / \mathrm{vaso}) \\
\text { Root dry matter }(g / \text { pot })\end{array}$ \\
\hline $\begin{array}{l}\text { Inoculante } 1 \\
\text { Inoculant 1 }\end{array}$ & $0,04 \mathrm{a}$ & $6,81 \mathrm{a}$ \\
Inoculante 2 & $0,05 \mathrm{a}$ & $7,15 \mathrm{a}$ \\
$\begin{array}{l}\text { Inoculant } 2 \\
\mathrm{TN}\end{array}$ & $0,02 \mathrm{~b}$ & $6,95 \mathrm{a}$ \\
$C N$ & $0,01 \mathrm{~b}$ & $4,83 \mathrm{~b}$ \\
$\mathrm{~T}$ & & \\
C
\end{tabular}

As médias nas colunas, seguidas por letras iguais, não diferem significativamente pelo teste LSD (5\%).

Means, within a column, followed by the same letters do not differ by LSD test $(5 \%)$.

TN (testemunha nitrogenada); T (testemunha sem nitrogênio). CN (control with nitrogen); C (control without nitrogen).

seca da parte aérea (MSPA) e teor de nitrogênio (N\%) indicaram que os efeitos de corte e da interação corte $\mathrm{x}$ tratamentos de inoculação foram significati$\operatorname{vos}(\mathrm{P}<0,01)$, de modo que o efeito de tratamentos de inoculação (TI) foi significativo apenas para N\% $(\mathrm{P}<0,01)$ (Tabela 2).

Os resultados (Tabelas 1 e 2) indicam que os dois inoculantes comerciais testados foram eficientes no desenvolvimento das três cultivares de alfafa em solo de cerrado com $\mathrm{pH}$ corrigido. $\mathrm{Na}$ Austrália, estudos sobre a eficiência de inoculantes no desenvolvimento de espécies de alfafa, incluindo a $M$. sativa $\mathrm{L}$., foram conduzidos por Howieson et al. (2000). Quando comparadas ao tratamento não-inoculado, todas as estirpes testadas foram capazes de proporcionar maiores produções de MSPA, no entanto, um dos inoculantes comerciais foi pouco eficiente na fixação de $\mathrm{N}_{2}$, produzindo apenas $53,8 \%$ do tratamento adubado com nitrogênio mineral (Howieson et al., 2000).

A eficiência dos inoculantes 1 e 2 também é comprovada pelos valores encontrados para $\mathrm{N} \%(2,52$ e 2,70, respectivamente) e N-total $(0,04$ e $0,05 \mathrm{~g} /$ vaso), que foram bem superiores aos obtidos nos tratamentos sem inoculantes, que apresentaram médias de 1,26 para N\% e 0,015 g/vaso de N-total.

Deve-se ressaltar que, no primeiro corte, a testemunha sem inoculante e sem nitrogênio foi similar aos cultivares dos demais tratamentos, provavelmente em 
Tabela 2 - Produção de massa seca da parte aérea (MSPA) e porcentagem de nitrogênio (N\%) obtidas com o uso ou não de inoculantes comerciais e adubação nitrogenada em alfafa, avaliados em dois cortes

Table 2 - Dry matter yield of the aboveground parts (DMAP) and nitrogen percentage (N\%), obtained with the use or not of commercial inoculants and nitrogen fertilization of alfalfa, in two harvests

\begin{tabular}{|c|c|c|c|c|}
\hline \multirow[t]{2}{*}{$\begin{array}{l}\text { Tratamento } \\
\text { Treatment }\end{array}$} & \multicolumn{2}{|c|}{$\begin{array}{c}\text { MSPA (g/vaso) } \\
\text { DMAP (g/pot) }\end{array}$} & \multicolumn{2}{|c|}{$\begin{array}{l}\mathrm{N}(\%) \\
N(\%)\end{array}$} \\
\hline & $\begin{array}{c}\text { Corte } 1 \\
\text { Harvest } 1 \\
\end{array}$ & $\begin{array}{c}\text { Corte } 2 \\
\text { Harvest } 2\end{array}$ & $\begin{array}{c}\text { Corte } 1 \\
\text { Harvest } 1\end{array}$ & $\begin{array}{c}\text { Corte } 2 \\
\text { Harvest } 2\end{array}$ \\
\hline $\begin{array}{l}\text { Inoculante } 1 \\
\text { Inoculant } 1\end{array}$ & $2,61 \mathrm{bA}$ & $1,84 \mathrm{aB}$ & $3,10 \mathrm{aA}$ & $2,52 \mathrm{aB}$ \\
\hline $\begin{array}{l}\text { Inoculante } 2 \\
\text { Inoculant } 2\end{array}$ & $3,04 \mathrm{abA}$ & $1,96 \mathrm{aB}$ & $3,14 \mathrm{aA}$ & $2,70 \mathrm{aB}$ \\
\hline $\begin{array}{l}\mathrm{TN} \\
C N\end{array}$ & $3,33 \mathrm{aA}$ & $1,5 \mathrm{abB}$ & $3,03 \mathrm{aA}$ & $1,43 \mathrm{bB}$ \\
\hline $\begin{array}{l}\mathrm{T} \\
C\end{array}$ & $3,00 \mathrm{abA}$ & $1,23 \mathrm{bB}$ & $2,84 \mathrm{aA}$ & $1,10 \mathrm{bB}$ \\
\hline
\end{tabular}

Médias nas colunas seguidas por letras minúsculas iguais não diferem $(P<0,05)$ entre si pelo teste LSD.

Médias do primeiro e segundo cortes seguidas por letras maiúsculas iguais não diferem $(P<0,01)$ entre si pelo teste $F$, dentro de linhas.

TN (testemunha nitrogenada); T (testemunha sem nitrogênio).

Means of first and second harvest followed by the same capital letters do not differ by $F$ test $(P<.01)$, within a row.

Means, within a column, followed by the same small letters do not differ $(P<.05)$ by $L S D$ test.

CN (control with nitrogen); C (control without nitrogen).

decorrência da mineralização do nitrogênio existente no solo, que foi suficiente para o desenvolvimento inicial das plantas. Esse processo é acentuado quando o solo é revolvido, e, principalmente quando da aplicação de calcário e fertilizantes. No segundo corte, porém, os dados de $\mathrm{N} \%$ obtidos com a testemunha nitrogenada que recebeu o correspondente a $20 \mathrm{~kg} / \mathrm{ha}$ de $\mathrm{N}$ semanalmente indicam que essa dose não foi suficiente para garantir um crescimento satisfatório, o que refletiu em valores mais baixos que os obtidos nos tratamentos inoculados.

Verifica-se também que, no primeiro corte, os valores encontrados para MSPA e N\% foram maiores que os obtidos no segundo corte (Tabela 2). Resalta-se que, além do efeito da mineralização do $\mathrm{N}$ abordado anteriormente, as diferenças nos intervalos de corte, o primeiro com 60 dias e o segundo com 30 dias, podem ter contribuído para estes resultados.

Com relação ao parâmetro nodulação, os tratamentos TN e T não nodularam, indicando que, no solo trabalhado, não há presença de $R$. meliloti na população nativa de rizóbio. Estas observações reforçam a necessidade de se inocular a alfafa com rizóbio específico nas regiões tropicais. Há indícios de que a introdução de estirpes de rizóbio torna-se mais difícil em solos que apresentam alta população nativa (Herridge \& Roughley, 1975; Brockwell et al., 1982). A ausência de estirpes específicas para alfafa nos solos tropicais propicia maior possibilidade de sucesso com a inoculação. No entanto, para aumentar o potencial de produção da cultura da alfafa, são necessários estudos mais detalhados sobre sua nodulação e fixação de nitrogênio atmosférico nas regiões tropicais. Franco (1994) ressaltou, em pesquisas anteriores, a importância desses estudos, mencionando a escassez de informações sobre os fatores limitantes da nodulação, simbiose e fixação de nitrogênio da alfafa nas condições brasileiras.

Destaca-se também a importância de trabalhos de longa duração, para verificar a eficiência da fixação de $\mathrm{N}_{2}$ no suprimento do nitrogênio, após pastejo ou corte da alfafa. Segundo Bélanger \& Richards (2000), a fixação de $\mathrm{N}_{2}$ na alfafa não foi suficiente para assegurar taxas de crescimento satisfatórias após desfolhação, recomendando-se a aplicação de fertilizantes nitrogenados para assegurar estabilidade de produção de forragem desta cultura. É recomendável, portanto, repetir o experimento em campo, com longa duração, para validação destes resultados. 


\section{Conclusões}

Em casa de vegetação, os inoculantes comerciais disponíveis no mercado foram eficientes na nodulação e no desenvolvimento da alfafa em solo de cerrado.

A população autóctone de rizóbio do Latossolo Vermelho-Escuro, fase cerrado, não foi eficiente na nodulação de alfafa, tornando-se necessária a inoculação com Rhizobium meliloti.

\section{Literatura Citada}

BÉLANGER, G.; RICHARDS, J.E. Dynamics of biomass and N accumulation of alfalfa under three $\mathrm{N}$ fertilization rates. Plant and Soil, v.219, p.177-185, 2000.

BOTREL, M.A.; ALVIM, M.J. Avaliação de cultivares de alfafa na Zona da Mata de Minas Gerais. Pesquisa Agropecuária Brasileira, v.32, n.9, p.971-975, 1997.

BOTREL, M.A.; FERREIRA, R.P.; CRUZ, C.D. et al. Estimativas de coeficientes de repetibilidade para produção de matéria seca em cultivares de alfafa sob diferentes ambientes. Revista Ceres, v.47, n.274, p.651-663, 2000.

BOTREL, M.A.; FERREIRA, R.P.; ALVIM, M.J. et al. Cultivares de alfafa em área de influência da Mata Atlântica no Estado de Minas Gerais. Pesquisa Agropecuária Brasileira, v.36, n.11, p.1437-1442, 2001.

BREMNER, J.M. Total nitrogen. In: BLACK, C.A. (Ed.) Methods of soil analysis. Madison: American Society of Agronomy, 1965. p.1149-1178 (Agronomy, 9).

BROCKWELL, J.; GAULT, R.R.; ZORIN, M. et al. Effects of environmental variables on the competition between inoculum strains and naturalized populations of Rhizobium trifolli for nodulation of Trifolium subterraneum L. and on rhizobia persistence in the soil. Australian Journal of Agricultural Research, v.33, p.803-815, 1982.

CARVALHO, J.G.; ASSIS, R.P.; MOREIRA, A. Necessidade de calagem para a cultura da alfafa. In: BOTREL, M.A.; ALVIM, M.J.; PASSOS, L.P. et al. (Eds.) Workshop sobre potencial forrageiro da alfafa (Medicago sativa) nos trópicos. Juiz de Fora: Embrapa Gado de Leite, 1994. p.127-132.

CARVALHO, L.A.; VILELA, D. Produção artificial de feno de alfafa (Medicago sativa L.) e seu uso na alimentação animal. In: CARVAlHO, L.A.; VILELA, D. (Eds.) Cultura da alfafa: estabelecimento, fenação, custo de produção e construção de um secador estático. Coronel Pacheco: Embrapa Gado de Leite, 1994. p.13-20.

CHENG, Y.; WATKIN, E.L.J.; O'HARA G.W. et al. Medicago sativa and Medicago murex differ in the nodulation response to soil acidity. Plant and Soil, v.238, p.31-39, 2002.

FERREIRA, R.P.; BOTREL, M.A.; PEREIRA, A.V. et al. Avaliação de cultivares de alfafa e estimativas de repetibilidade de caracteres forrageiros. Pesquisa Agropecuária Brasileira, v.34, n.1, p.995-1002, 1999.

FRANCO, A. A. Nutrição nitrogenada da alfafa em solos tropicais. In: BOTREL, M.A.; ALVIM, M.J.; PASSOS, L.P. et al. (Eds.) Workshop sobre potencial forrageiro da alfafa (Medicago sativa) nos trópicos. Juiz de Fora: EMBRAPACNPGL, 1994. p.127-132.

HERRIDGE, B.F.; ROUGHLEY, R.J. Nodulation of and nitrogen fixation by Lablab purpureus under field conditions. Australian Journal of Experimental Agriculture and Animal Husbandry, v.15, p.264-269, 1975.

HIECHEL, G.H.; BARNES, D.K.; HENJUM, K.I. N2 fixation, and $\mathrm{N}$ and dry matter partitioning during a 4-year alfalfa stand. Crop Science, v.24, p.811-815, 1984.

HOWIESON, J.G.; NUTT, B.; EVANS, P. Estimation of hoststrain compatibility for symbiotic $\mathrm{N}$-fixation between Rhizobium meliloti, several annual species of Medicago and Medicago sativa. Plant and Soil, v.219, p.49-55, 2000.

MOREIRA, A.; EVANGELISTA, A.R.; RODRIGUES, G.H.S. Avaliação de cultivares de alfafa na região de Lavras, Minas Gerais. Pesquisa Agropecuária Brasileira, v.31, n.6, p.407-411, 1996.

PEREIRA, A.V.; FERREIRA, R.P.; CRUZ, C.D. et al. Comportamento de alfafa cv. crioula de diferentes origens e estimativas dos coeficientes de repetibilidade para caracteres forrageiros. Revista Brasileira de Zootecnia, v.27, n.4, p.686-690, 1998.

PEREIRA, A.V.; VALLE, C.B.; FERREIRA, R.P. et al. Melhoramento de forrageiras tropicais. In: NASS, L.L.; VALOIS, A.C.C.; MELO, I.S. et al. (Eds.) Recursos genéticos e melhoramento de plantas. Rondonópolis: Fundação Mato Grosso, 2001. p.549-602.

RAMALHO, M.A.P.; FERREIRA, D.F.; OLIVEIRA, A.C. Experimentação em genética e melhoramento de plantas. Lavras: Universidade Federal de Lavras, 2000. 326p.

SALES, E.C.J. Produtividade, composição bromatológica e degradabilidade ruminal de cultivares de alfafa (Medicago sativa L.). Lavras: Universidade Federal de Lavras, 2001. 105p. Tese (Doutorado em Zootecnia) - Universidade Federal de Lavras, 2001.

SIQUEIRA, C.; CARVALHO, M.M.; SARAIVA, O.F. et al. Resposta de três gramíneas forrageiras tropicais à aplicação de calcário e fósforo em um solo ácido. In: CONGRESSO BRASILEIRO DE ZOOTECNIA, 1., 1980, Fortaleza. Anais... Fortaleza: Universidade Federal de Fortaleza, 1980. p.473.

VIANA, M.C.M.; KONZEN, E.; PURCINIO, H.M.A. Comportamento de 28 cultivares de alfafa nas condições de cerrado de Sete Lagoas. In: REUNIÃO ANUAL DA SOCIEDADE BRASILEIRA DE ZOOTECNIA, 35., 1998, Botucatu. Anais... Botucatu: Sociedade Brasileira de Zootecnia, 1999. 1 CD.

XAVIER, D.F.; CARVALHO, M.M. Inoculação de leguminosas. Coronel Pacheco, MG: Embrapa Gado de Leite, 1985. p. 1-17. (Circular Técnica, 24)

Recebido em: 04/08/04 Aceito em: 29/01/05 